\title{
Characterization Study of PVDF/ Cloisite Hollow Fiber Membrane in Aqueous Diethanolamine
}

\author{
R. Naim ${ }^{1,2^{*}}$, S. Abdullah ${ }^{1,2}$, N. Zulkifli ${ }^{1}$ \\ ${ }^{1}$ Faculty of Chemical \& Natural Resources Engineering, Lebuhraya Tun Razak, \\ 26300 Gambang, Kuantan, Pahang \\ ${ }^{2}$ Center of Excellence for Advanced Research in Fluid Flow (CARIFF), Lebuhraya \\ Tun Razak, 26300 Gambang, Kuantan, Pahang
}

\begin{abstract}
Different concentrations of PVDF/Cloisite hollow fiber membranes were prepared and studied via immersion test. The differences in membrane properties were observed by immersion tests at 10 days, 20 days and 30 days in DEA solution. The membranes were characterized via liquid entry pressure $\left(\mathrm{LEP}_{\mathrm{w}}\right)$ and contact angle. Surface morphology analysis study was conducted before and after immersion test using scanning electron microscopy. From the result obtained, the liquid entry pressure and contact angle showed decreasing effect after immersion in DEA solution for 30 days. SEM analysis indicated that increasing DEA concentration creates more finger-like structure compared to pristine membrane. Incorporation of cloisite into PVDF membranes improves the contact angle value but development of more finger-like structure is subjected to wetting problem.
\end{abstract}

Keywords: PVDF, Cloisite, hollow fiber membrane contactor, diethanolamine

\subsection{INTRODUCTION}

Carbon dioxide is the most known greenhouse gas and is responsible for two-thirds of the greenhouse effects [1]. One of the methods used to separate $\mathrm{CO}_{2}$ gas from the flue gas is membrane contactor technology. Lv et al. [2] described membrane contactor as a hybrid technology that combines the advantages of chemical (high selectivity) and membrane (modularity and compact structure) absorption. In the application of membrane contactor for removing $\mathrm{CO}_{2}$, it is very important to consider the effects of membrane materials on the $\mathrm{CO}_{2}$ absorption performance via the compatibility of the polymer material with liquid absorbent. Selection of proper polymeric materials for membrane gas absorption is of much concern as the main criteria for polymer materials should be hydrophobic. This is to reduce the effects of wetting on the membrane pores by providing efficient mass transfer between gas and liquid phase.

A study performed by Mansourizadeh and Ismail [3, 4] indicated that the addition of different additives exhibit good $\mathrm{CO}_{2}$ absorption performance. Liu et al., [5] has employed polyvinylidene fluoride (PVDF) polymer for hollow fiber membrane contactor due to its solubility in almost all organic solvent. In addition, PVDF also possess properties such as high thermal and chemical resistant, high mechanical strength and abrasion resistance. Incorporation of inorganic filler cloisite clay into PVDF membrane matrix has been discussed by Rezaei et al. [6]. It was found that the permeation flux increased significantly

* Corresponding to: R. Naim (email: rosmawati@ump.edu.my) 
with the increasing cloisite ratio thus improved the wetting resistance of membranes in terms of contact angle and liquid entry pressure.

In order to address the membrane liquid absorbent compatibility issue, Lv et al. [2] performed wettability study on commercial polypropylene hollow fiber membrane in two different amine solutions, namely monoethanolamine (MEA) and methyldiethanolamine (MDEA). It was observed that the membrane suffers pore wetting due to alteration of surface properties and reduction of membrane hydrophobicity. This is in agreement with the finding by Wang et al. [7] where Celgard X40-200 and Celgard X50-215 hollow fiber membrane showed decreasing contact angle value after immersion in diethanolamine (DEA) solution. This can be verified by the XPS analyses indicating the possible chemical reactions have taken place between the membranes and DEA which may be responsible for the decreasing contact angle values. This study mainly focussed on the compatibility of PVDF/cloisite hollow fiber membrane in DEA solution. Characterization were conducted to measure the liquid entry pressure and contact angle while scanning electron microscopy (SEM) was used to obtain surface micrograph of the membrane before and after immersion in amine solution for 30 days.

\subsection{EXPERIMENTAL}

\subsection{Materials}

PVDF polymer (Kynar 740) was purchased from Arkema Inc., USA in the form of pellets, non-solvent additive - Cloisite ${ }^{\circledR} 15 \mathrm{~A}, \quad \mathrm{~N}$ methylpyrrolidone (NMP) was used as solvent without further purification.
Diethanolamine (DEA, > 99\%) from Merck was used as liquid absorbent. Methanol (GR grade, 99.9\%) and nhexane $(99 \%)$ were used as posttreatment solution for the prepared membranes.

\subsection{Fabrication of Hollow Fiber Membranes}

The polymer was dried in a vacuum oven over $24 \mathrm{~h}$ periods at $60^{\circ} \mathrm{C}$ to remove moisture content. The PVDF polymer was weighed and $15 \%$ of polymer solution dope was prepared with various $\% \mathrm{wt}$ concentration of cloisite $(1 \%, 3 \%$ \& 5\%). After the polymer dope was continuously stirred at $60{ }^{\circ} \mathrm{C}$, the solution was degassed using ultrasonic bath for 1 hour to eliminate air bubbles. The spun fibers were fabricated using spinning rig with specific spinning condition. The fibers were then post-treated with methanol and n-hexane to minimize fiber shrinkage and pore-collapse prior to drying at room temperature.

\subsection{Immersion Test}

The PVDF hollow fiber membranes were immersed in $10 \%$ wt. DEA aqueous solution. Two large containers were prepared for this purpose and each container kept 20 hollow fiber membranes with different cloisite concentrations. The fibers were left immersed in the aqueous solution for 30 days. Each fibers were then taken out for characterization after immersion for 10 days, 20 days and 30 days. Prior to characterization test, five pieces of fibers were taken out and washed with deionized water for several times. Subsequently, the fibers were dried to eliminate the remnant amine absorbent on the fiber surface. 


\subsection{Liquid Entry Pressure (LEPw)}

Liquid entry pressure $\left(\mathrm{LEP}_{\mathrm{W}}\right)$ was measured using a test module consist of one or two fibers where deionized water was fed into the lumen side of the fiber using a diaphragm pump. Then, the pressure was slowly increased at set intervals starting at 0.5 bars. The fibers were kept at constant pressure at each pressure interval for about 20 minutes to observe on the appearance of first water droplets at the outer surface of the membranes. The LEP ${ }_{\mathrm{W}}$ value was considered when the first water droplet appears at the outer membrane surface. The LEPw value reported here is taken from the average value of five recorded readings.

\subsection{Contact Angle Measurements}

A contact angle goniometer (OCA15plus, DataPhysics), equipped with image-processing software was used to evaluate the degree of membrane hydrophobicity via sessile drop technique. Ten spots were measured on each sample and averaged.

\subsection{Scanning Electron Microscopy (SEM) Analysis}

The morphology of hollow fiber membranes are analyzed using scanning electron microscopy (SEM) (Philips XL40). The samples are gently fractured in liquid nitrogen prior to platinum coating. The cross section structures for both outer and inner skin layer of the spun fibers were taken at various magnifications.

\subsection{RESULTS AND DISCUSSION}

\subsection{Liquid Entry Pressure (LEPw) Test Results}

Table 1 and Table 2 show the liquid entry pressure of water of PVDF/Cloisite hollow fiber membranes before and after the immersion test. Liquid entry pressure (LEPw) is defined as the pressure difference from which the liquid penetrates into membrane pores and the first water droplets that appear on the outlet of dry fiber skin. The pressure difference is correlated to the interfacial tension, the contact angle of the liquid on the membrane surface and pore size of the membrane. Theoretically, the addition of Cloisite in the membrane significantly improved the membrane characteristics in terms of wetting pressure, hydrophobicity and mean pore size. This might indicate that the arrangement of polymer chain packing has been disordered by the presence of clay particles in the membrane matrix contributing to better structural properties. The higher liquid entry pressure value obtained by the composite membranes showed that the membranes are able to prevent liquid penetration through the membrane pores due to the cross-membrane pressure difference that is sometimes caused by imbalance of feed and permeate side pressures. From the results obtain, Sample 4 possess the best attributes in preventing liquid penetration. 
Table 1 Liquid entry pressure of PVDF/Cloisite membranes

\begin{tabular}{ccccc}
\hline & \multicolumn{4}{c}{ Pressure (bar) } \\
\cline { 2 - 5 } Sample & 1st & 2nd & 3rd & $\begin{array}{c}\text { Average } \\
\text { value }\end{array}$ \\
\hline S1 & 6 & 5.5 & 5 & $5.5 \pm 4.1$ \\
S2 & 3 & 3.5 & 4.4 & $3.6 \pm 3.5$ \\
S3 & 4 & 4.5 & 4 & $4.2 \pm 2.1$ \\
S4 & 6 & 6.5 & 7 & $6.5 \pm 1.3$ \\
\hline
\end{tabular}

The immersed membranes show a decreasing value of liquid entry pressure. This finding is in agreement with Wang et al. (2004) as stated before, where the immersions of hollow fiber membranes cause a change on the surface morphology.

Table 2 Liquid entry pressure of PVDF/Cloisite membranes after immersion test

\begin{tabular}{ccccc}
\hline \multirow{2}{*}{ Molarity } & & \multicolumn{3}{c}{ Pressure, Bar } \\
\cline { 3 - 5 } & Sample & $\begin{array}{c}\mathbf{1 0} \\
\text { days }\end{array}$ & $\begin{array}{c}\mathbf{2 0} \\
\text { days }\end{array}$ & $\begin{array}{c}\mathbf{3 0} \\
\text { days }\end{array}$ \\
\cline { 3 - 5 } & & \multicolumn{3}{c}{ Average value } \\
\hline \multirow{4}{*}{$1 \mathrm{M}$} & $\mathrm{S} 1$ & 4.8 & 3.15 & 2.5 \\
& $\mathrm{~S} 2$ & 2.7 & 2.5 & 1.5 \\
& $\mathrm{~S} 3$ & 2.5 & 1.75 & 1.5 \\
& $\mathrm{~S} 4$ & 1.5 & 2.85 & 1.5 \\
\hline \multirow{4}{*}{$2 \mathrm{M}$} & $\mathrm{S} 1$ & 3.55 & 4 & 1.5 \\
& $\mathrm{~S} 2$ & 2.25 & 1.75 & 1.75 \\
& $\mathrm{~S} 3$ & 2.75 & 2 & 1.75 \\
& $\mathrm{~S} 4$ & 1.5 & 2.5 & 1.5 \\
\hline
\end{tabular}

The data from the liquid entry pressure of the immersed membranes shows that Sample 1, the pristine membrane had the highest liquid entry pressure in both concentration and all of immersion durations. This might be caused by the morphology of the Cloisite particle contained in the other membrane samples due to the immersion in DEA. The Cloisite which reside in the inner surface morphed causing the spongy structure to fail to support the pressure introduced to the lumen side of the membranes.

\subsection{Contact Angle Measurements}

The addition of Cloisite also increased the contact angle of the PVDF/Cloisite membranes, which is mainly due to the higher surface roughness. The theory of Wenzel or Cassie effect explained this phenomenon that the presence of air pockets underneath the rough surface tends to hinder the liquid from penetrating into the grooves, which in turn increases surface hydrophobicity [8]. From the Table 3, there is no respective value that can correlate the contact angle value and the weight percent of Cloisite added. This result might be affected by other factors such as surface cleanliness during contact angle measurements.

Table 3 Contact angle measurement of PVDF/Cloisite membranes (before immersion)

\begin{tabular}{cc}
\hline Membrane & CA $\left(^{\circ}\right)$ \\
\hline S1 & $76.09 \pm 7.04$ \\
S2 & $87.21 \pm 2.26$ \\
S3 & $78.18 \pm 5.47$ \\
S4 & $77.22 \pm 5.54$ \\
\hline
\end{tabular}

Table 4 Contact angle measurement of PVDF/Cloisite membranes after immersion test

\begin{tabular}{ccc}
\hline \multirow{2}{*}{ Membrane } & \multicolumn{2}{c}{ Contact angle $\left(^{\circ}\right)$} \\
\cline { 2 - 3 } & 1M DEA & 2M DEA \\
\hline S1 & $75.01 \pm 4.67$ & $78.04 \pm 3.08$ \\
S3 & $82.69 \pm 3.51$ & $78.16 \pm 5.22$ \\
S4 & $83.44 \pm 3.44$ & $78.99 \pm 2.48$ \\
\hline
\end{tabular}

\subsection{Scanning Electron Microscopy (SEM) Analysis}

Figure 1 depicts the SEM micrographs for the cross-sectional image of 
PVDF/Cloisite hollow fiber membranes before the immersions. All of the micrographs show large fingerlike macrovoids stretched from the outer to the inner fiber skin. This explains the high value obtained in the liquid entry pressure before the immersion. The inner surface contained cloisite supported with large finger-like macrovoid produced a higher liquid entry pressure.
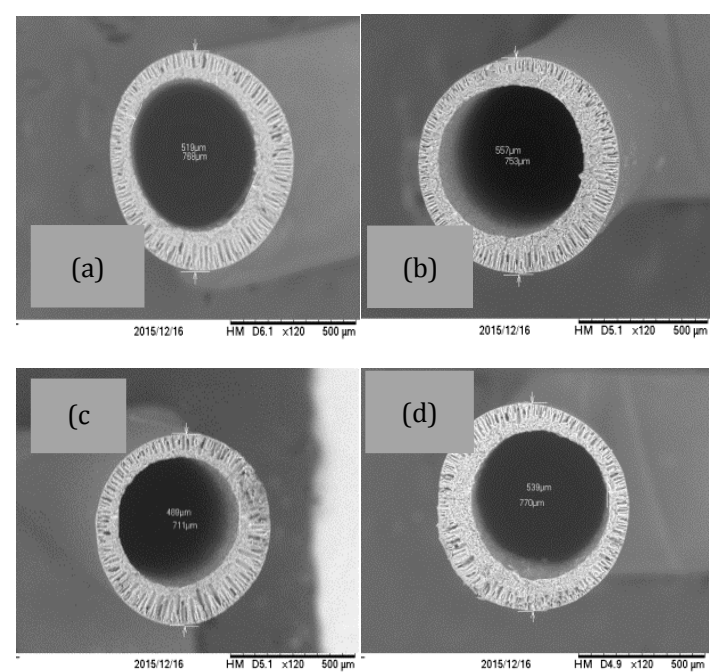

Figure 1 SEM micrographs of PVDF/Cloisite membranes, (a) PVDF, (b) 1.0 wt.\% Cloisite, (c) 3.0 wt.\% Cloisite and (d) 5.0 wt. $\%$ Cloisite

However, the immersed membranes shows that the macrovoid alone cannot support a high value of liquid entry pressure. On the bright side, the immersed membrane might prove that the macrovoid structure has effect on the surface roughness which lead to higher contact angle obtains from the immersed membrane. This is shown by the Sample 4 immersed in both concentration of DEA and all of the immerse duration. The sample Figure 2 c (i) and c (ii) show the appearance of new air pocket after the immersion. This is supported by the high contact angle measurement as shown in Table 4.
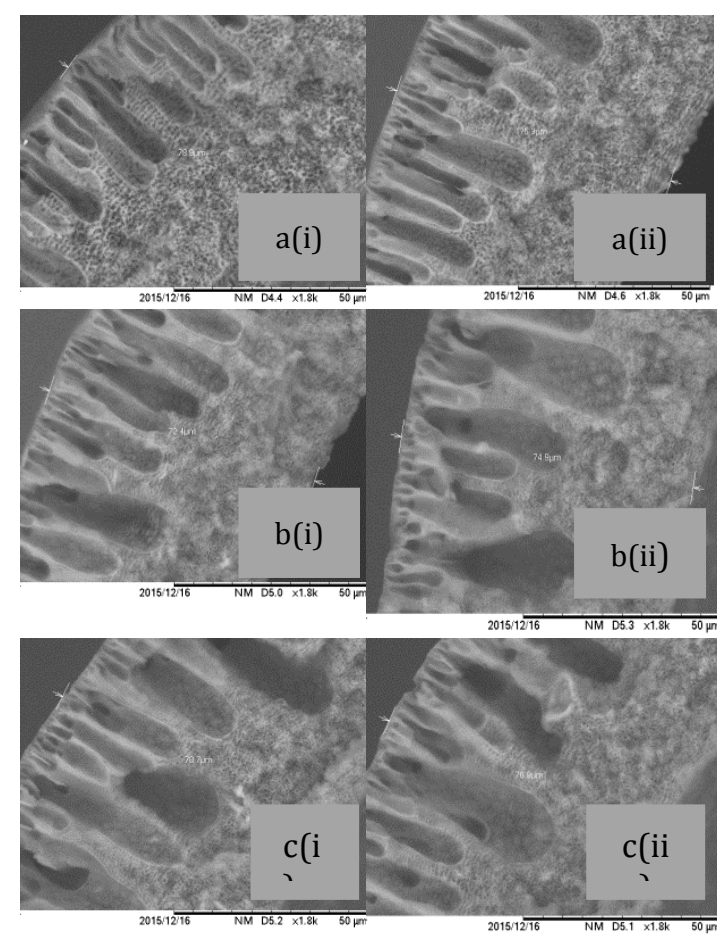

Figure 2 SEM micrographs of PVDF/Cloisite membranes, (a) 1MS1, (b) $1 \mathrm{MS} 4$ and (c) 2MS4; (i) 10 days and (ii) 30 days.

\subsection{CONCLUSION}

In this study, the addition of cloisite on the properties of PVDF membranes has been successfully prepared, characterized and evaluated. The cloisite clay present in the membrane contributed to a high liquid entry pressure in Sample 4. Cross-sectional SEM images revealed that cloisite addition has effect on the membrane internal, porosity and surface pore structure, irrespective of the weight percentage. In conclusion, cloisite clay in the PVDF membranes could improve the membrane structural properties. 


\section{ACKNOWLEDGEMENT}

The authors wish to thank the Universiti Malaysia Pahang (RDU140329) for the financial support through pursuing this research.

\section{REFERENCES}

[1] Zhang, H. Y., Wang, R., Liang D. T., Tay, J. H. 2008. Theoretical And Experimental Studies Of Membrane Wetting In The Membrane Gas-Liquid Contacting Process For $\mathrm{CO}_{2}$. Absorption Journal of Membrane Science. 308: 162-170.

[2] Lv, Y., Yua, X., Tua, S.T., Yan, J., Dahlquist, E. 2010. Wetting Of Polypropylene Hollow Fiber Membrane Contactors. Journal of Membrane Science. 362: 444452.

[3] Mansourizadeh, A. F. Ismail, M. S. Abdullah, B. C. Ng. 2010. Preparation of Polyvinylidene Fluoride Hollow Fiber Membranes for $\mathrm{CO}_{2}$ Absorption Using Phase-Inversion Promoter Additives. Journal of Membrane Science. 355: 200-207.

[4] Mansourizadeh, A., A. F. Ismail. 2011. Preparation And Characterization of Porous PVDF Hollow Fibre Membranes
For $\mathrm{CO}_{2}$ Absorption: Effect Of Different Non-Solvent Additives In The Polymer Dope. International Journal of Greenhouse Gas Control. 5: 640-648.

[5] Liu, F., Hashim, N. A., Liu, Y., Moghareh Abed, M. R., K. Li. 2011. Review Progress in The Production and Modification of PVDF Membranes. Journal of Membrane Science. 375: 1-27.

[6] Rezaei, M., Ismail, A. F., Bakeri, G. H., Hashemifard, S. A., Matsuura, T. 2015. Effect of General Montmorillonite and Cloisite 15A on Structural Parameters And Performance of Mixed Matrix Membranes Contactor for $\mathrm{CO}_{2}$ absorption. Chemical Engineering Journal. 260: 875-885.

[7] Wang, R., D. F. Li, D. T. Liang. 2004. Modeling Of $\mathrm{CO}_{2}$ Capture by Three Typical Amine Solutions in Hollow Fiber Membrane Contactors Chemical Engineering and Processing. 43: 849-856.

[8] Tai-Homgyoung, Leo-Wang Chen. 1995. Pore Formation Mechanism of Membranes from Phase Inversion Process. Desalination. 103: 233-247. 